\title{
Review
}

\section{Self-Management of Depression: \\ A Manual for Mental Health and Primary Care Professionals}

Albert Yeung, Greg Feldman \& Maurizio Fava Cambridge University Press, 2009, £30.00 pb, 216 pp. ISBN 9780521710084

With increasing pressures on healthcare costs, this book offers an approach to treating depressive disorders that will reduce the cost and potentially improve outcomes. Self-management, so far used in patients with diabetes and congestive heart disease, seems to lend itself well to the management of depression.

The evidence supporting self-management is reviewed, with an overview of the key principles: sharing decision-making with patients and promoting patients' self-efficiency. The authors go on to describe management of depression in primary care, followed by how self-management would benefit patients and reduce the burden on healthcare workers.

The bulk of the book covers suggestions of methods that can be used in self-management: self-assessment instruments, bibliotherapy, computer-based cognitive-behavioural therapy, meditation and exercise. Each chapter reviews the evidence available and suggests how to introduce each method into your practice. A number of examples of evidence-based assessment tools are given, along with reputable websites and books that can be used for self-management. The book describes how each tool can be used as an alternative or an adjunct to medication while still highlighting the importance of the doctor in supporting and treating depression. The authors examine overcoming barriers to using self-management in practice and applying it in a number of settings.

The review of the literature is thorough and the authors indicate the areas in which evidence is still lacking. It would have been helpful to have summary boxes or bullet-point lists in each chapter, so that having read the background one could quickly access the tools available in each chapter.

The book provides the clinician with essential information to practise evidence-based self-management. However, in its bid to comprehensively present the evidence, its usefulness as an instructive manual is somewhat diminished.

Floriana Coccia is Specialty Trainee 6, Birmingham and Solihull Mental Health NHS Foundation Trust, The Barberry Centre, Birmingham, email: floriana.coccia@yahoo.co.uk

doi: 10.1192/pb.bp.110.030429 